\title{
Spatially Confined Formation of Single Atoms in Highly Porous Carbon Nitride Nanoreactors
}

Yunpeng Zuo ${ }^{1}$, Tingting $\mathrm{Li}^{2}$, Ning Zhang ${ }^{1}$, Tianyun Jing ${ }^{3}$, Dewei Rao ${ }^{3 *}$, Patrik Schmuki ${ }^{4,5}$, Štěpán Kment ${ }^{5,6^{*}}$, Radek Zbořil ${ }^{5,6}$, Yang Chai ${ }^{{ }^{*}}$

${ }^{1}$ Department of Applied Physics, The Hong Kong Polytechnic University, Hung Hom, Kowloon, Hong Kong, People's Republic of China.

${ }^{2}$ Institute of Surface Micro and Nano Materials, Xuchang University, Xuchang, Henan 461002, People's Republic of China.

${ }^{3}$ School of Materials Science and Engineering, Jiangsu University, Zhenjiang 212013, People's Republic of China.

${ }^{4}$ Department of Materials Science, University of Erlangen-Nuremberg, Institute for Surface Science and Corrosion WW4-LKO, Martensstraße 7, D-91058 Erlangen, Germany.

${ }^{5}$ Regional Centre of Advanced Technologies and Materials, Czech Advanced Technology and Research Institute, Palacký University, Křŕžkovského 511/8, 77900 Olomouc, Czech Republic.

${ }^{6}$ CEET, Nanotechnology Centre, Centre of Energy and Environmental Technologies, VŠB-Technical University of Ostrava, 17. Listopadu 2172/15, 70800 Ostrava-Poruba, Czech Republic. 
Supplementary Figures

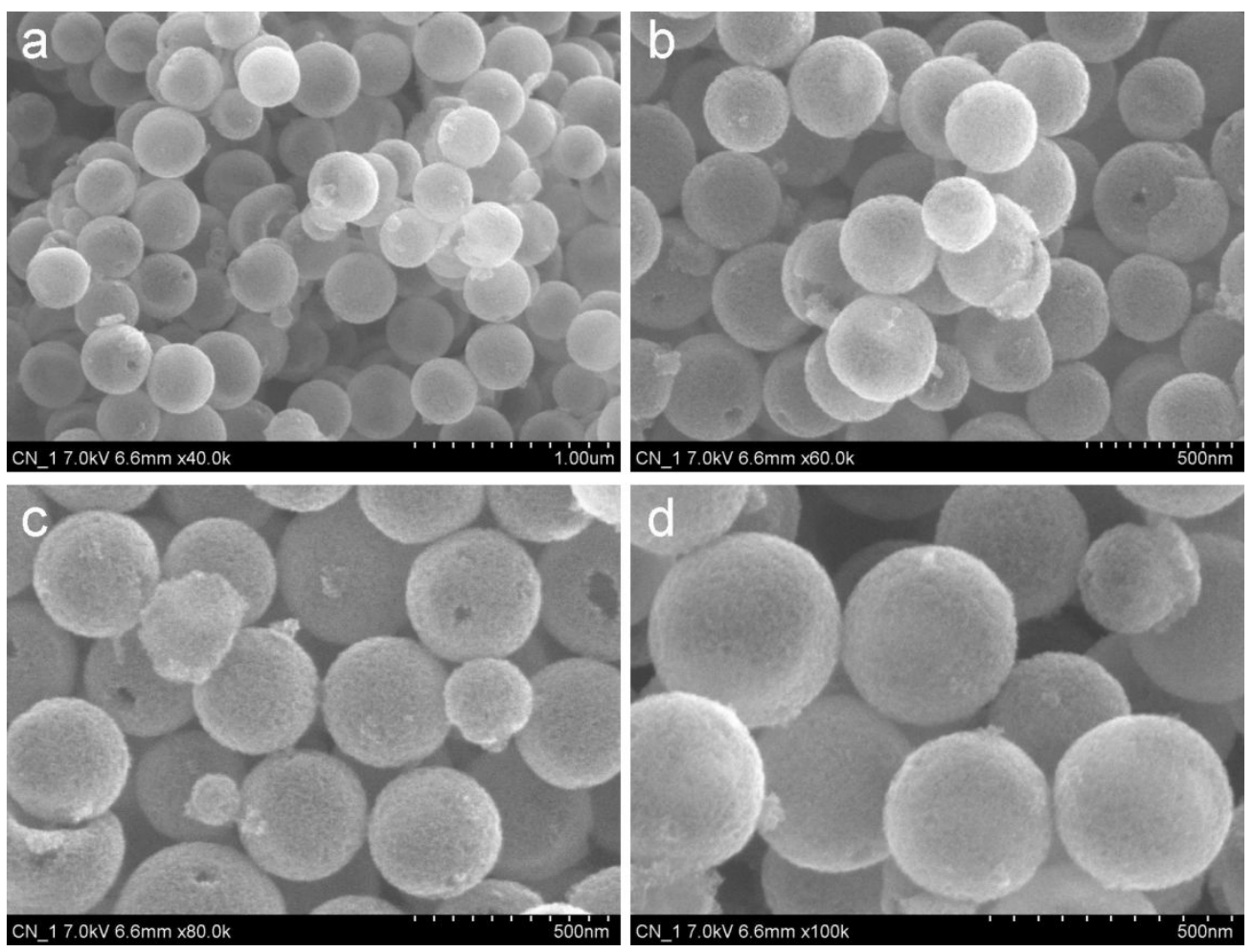

Figure S1. Morphological characterization of $p-C N$. a, b, c, d Enlarged SEM images of $p-\mathrm{CN}$. 

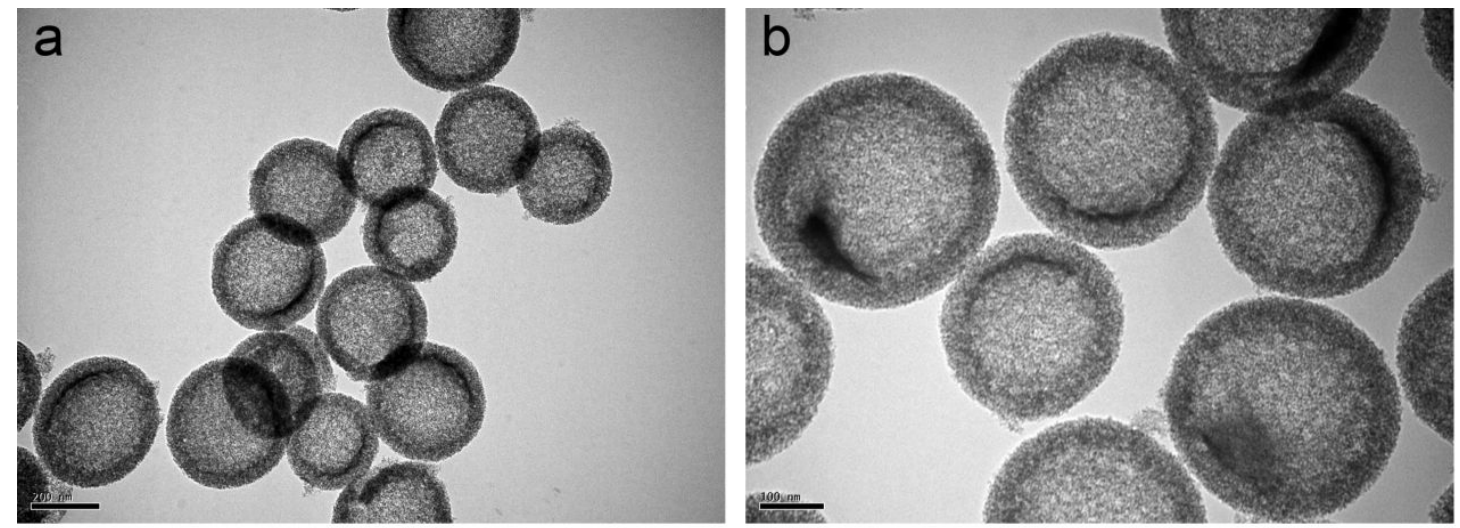

Figure S2. Structural characterization of $p$-CN. a, b Enlarged TEM images of $p$-CN. 

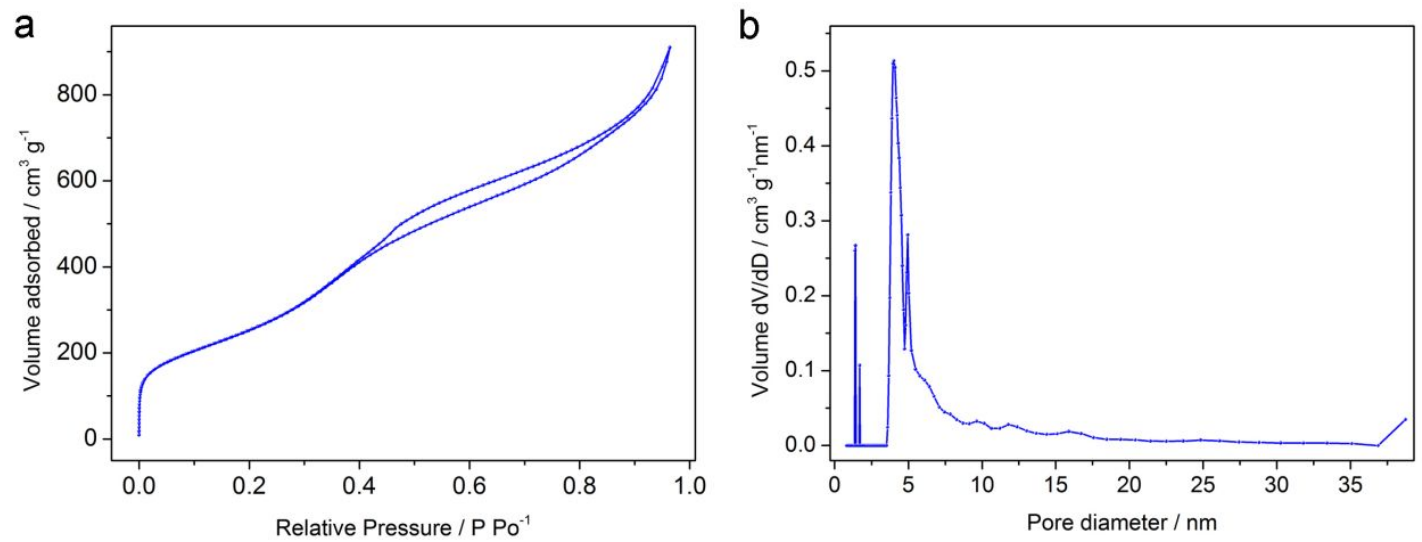

Figure S3. Characteristic analysis of specific surface area. a BET curves of the $p$-CN.

b The pore-size distribution of $p$-CN. 


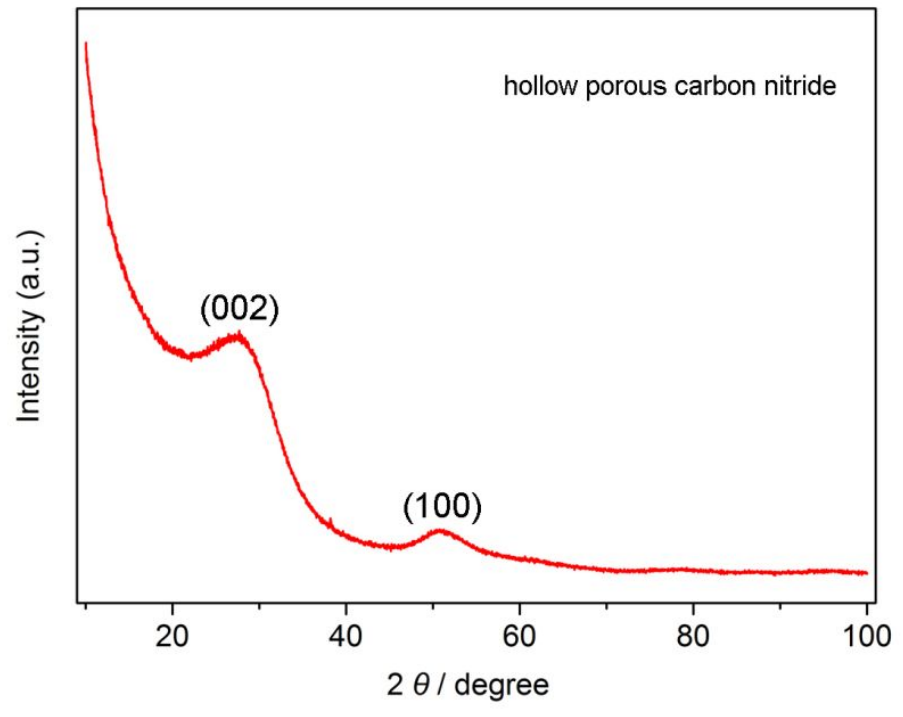

Figure S4. The XRD spectra of the $p-\mathrm{CN}$. 


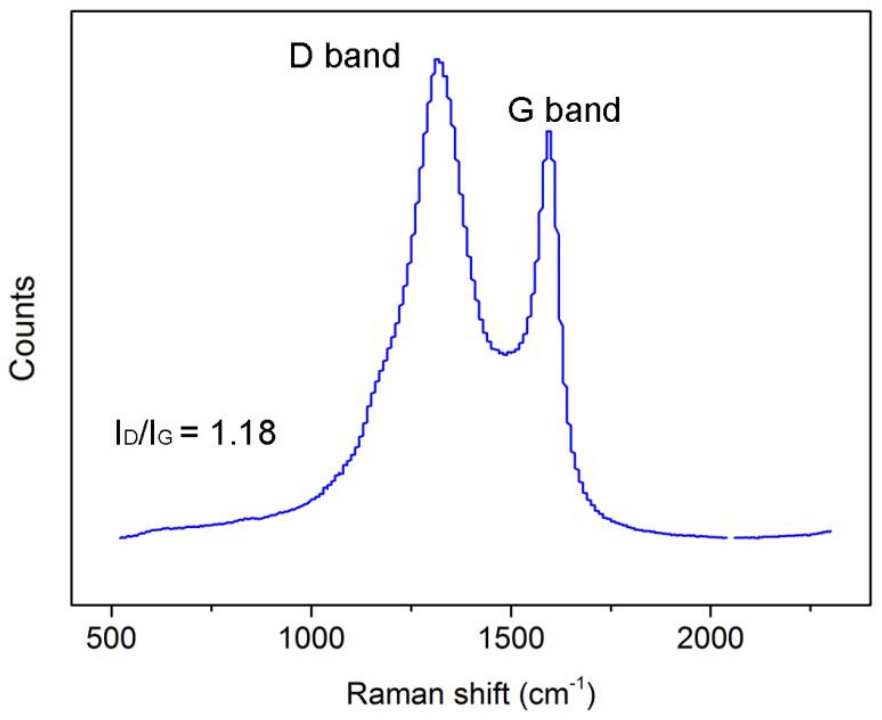

Figure S5. The Raman spectra of the $p-\mathrm{CN}$. 

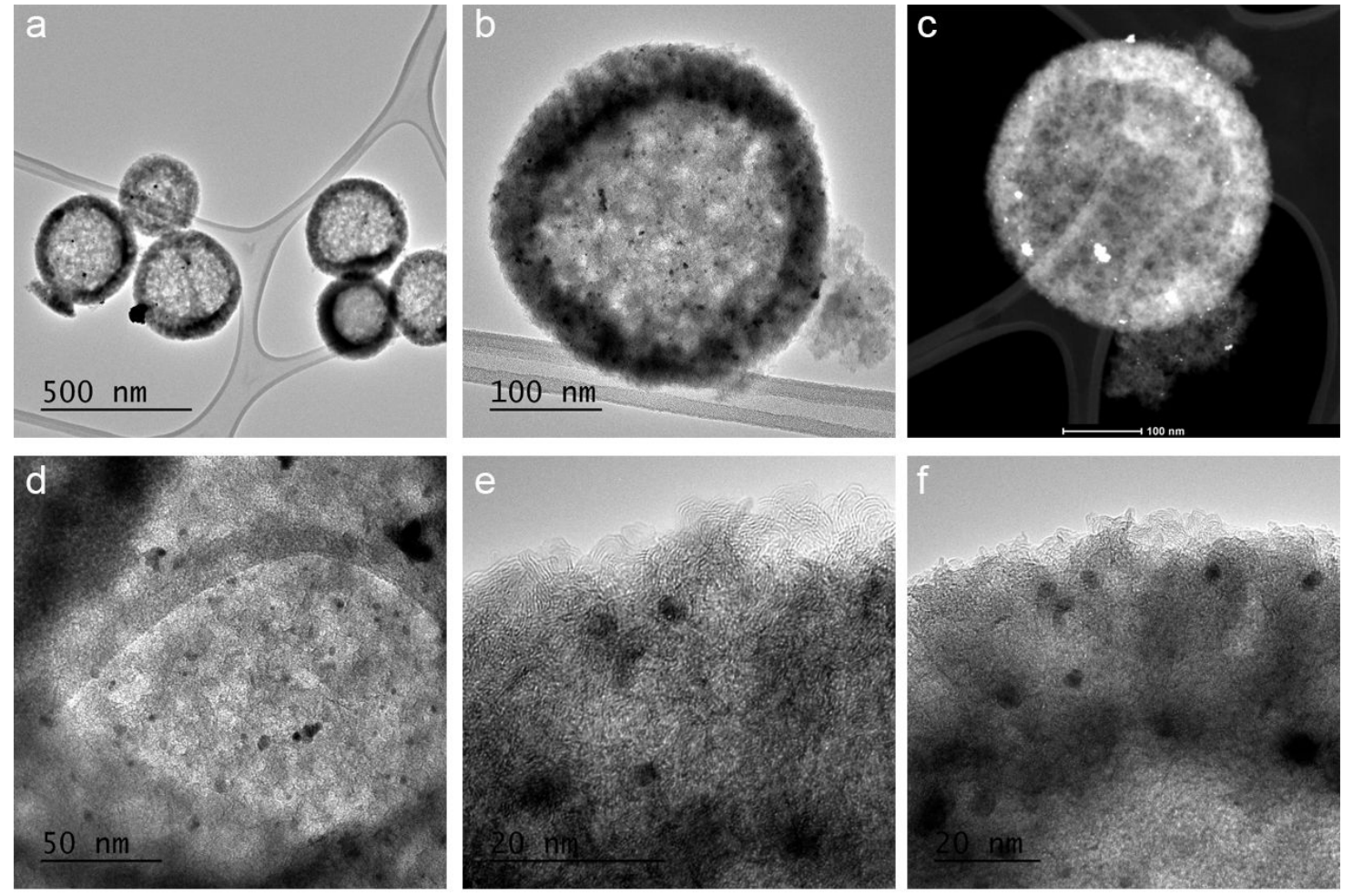

Figure S6. Structure analysis of TEM. a, b The enlarged TEM images of $p$-CN loaded Pt nanoparticles (PtNPs). c HAADF-STEM image of a typical $p$-CN sphere loaded PtNPs. d, e, f The HRTEM images of the $p$-CN loaded PtNPs reveal the PtNPs were embedded in the pore channels with some single Pt atoms. 


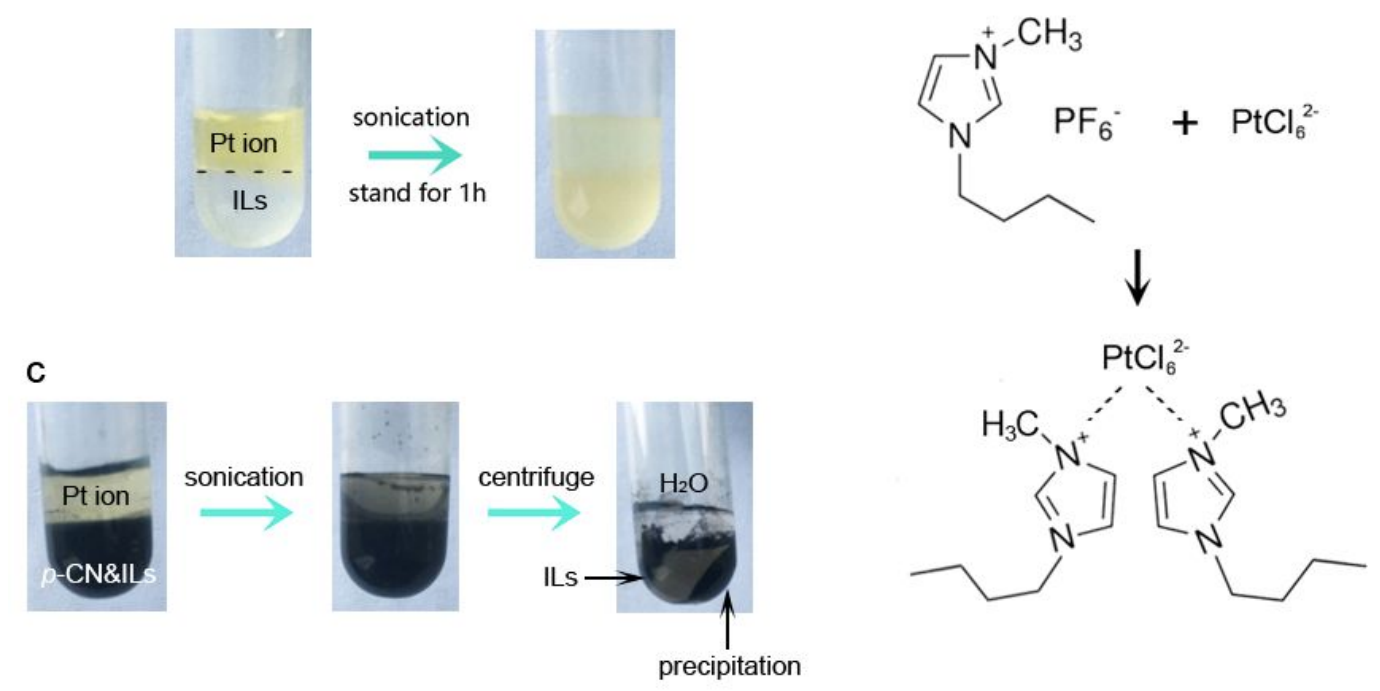

Figure S7. Typical process of experiment. a $\mathrm{C}_{8} \mathrm{H}_{15} \mathrm{~N}_{2} \mathrm{~F}_{6} \mathrm{P}-\mathrm{Pt}$ complexes will be generated after mixed $\mathrm{Pt}$ ion solution with $\mathrm{C}_{8} \mathrm{H}_{15} \mathrm{~N}_{2} \mathrm{~F}_{6} \mathrm{P}$. b The reaction process between $\mathrm{C}_{8} \mathrm{H}_{15} \mathrm{~N}_{2} \mathrm{~F}_{6} \mathrm{P}$ and $\mathrm{H}_{2} \mathrm{PtCl}_{6}$. c After centrifugation, a certain amount of $\mathrm{C}_{8} \mathrm{H}_{15} \mathrm{~N}_{2} \mathrm{~F}_{6} \mathrm{P}$-stabled Pt precursor were adsorbed on the surface of the $p$-CN. 


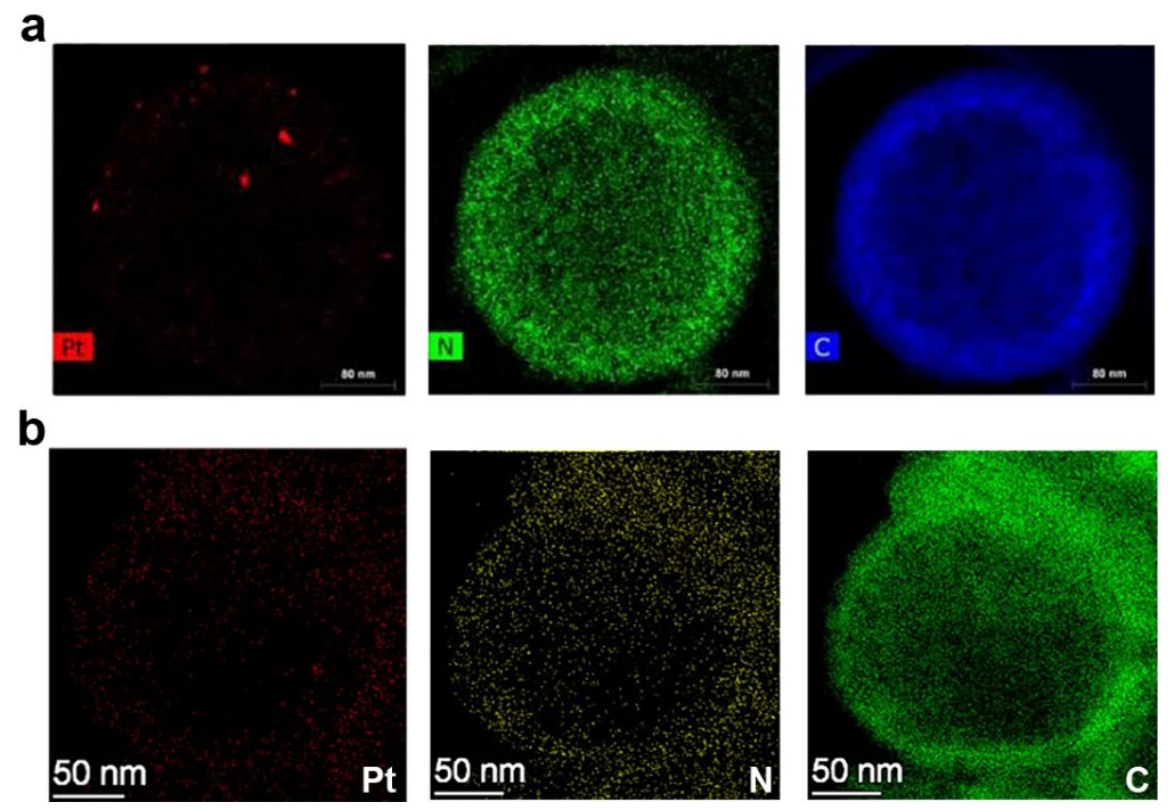

Figure S8. Characterization of the p-CN@Pt and p-CN@S-Pt. a,b EDS mapping images of the $p-\mathrm{CN} @ \mathrm{Pt}$ and $p$-CN@S-Pt correspond to Fig.1. 

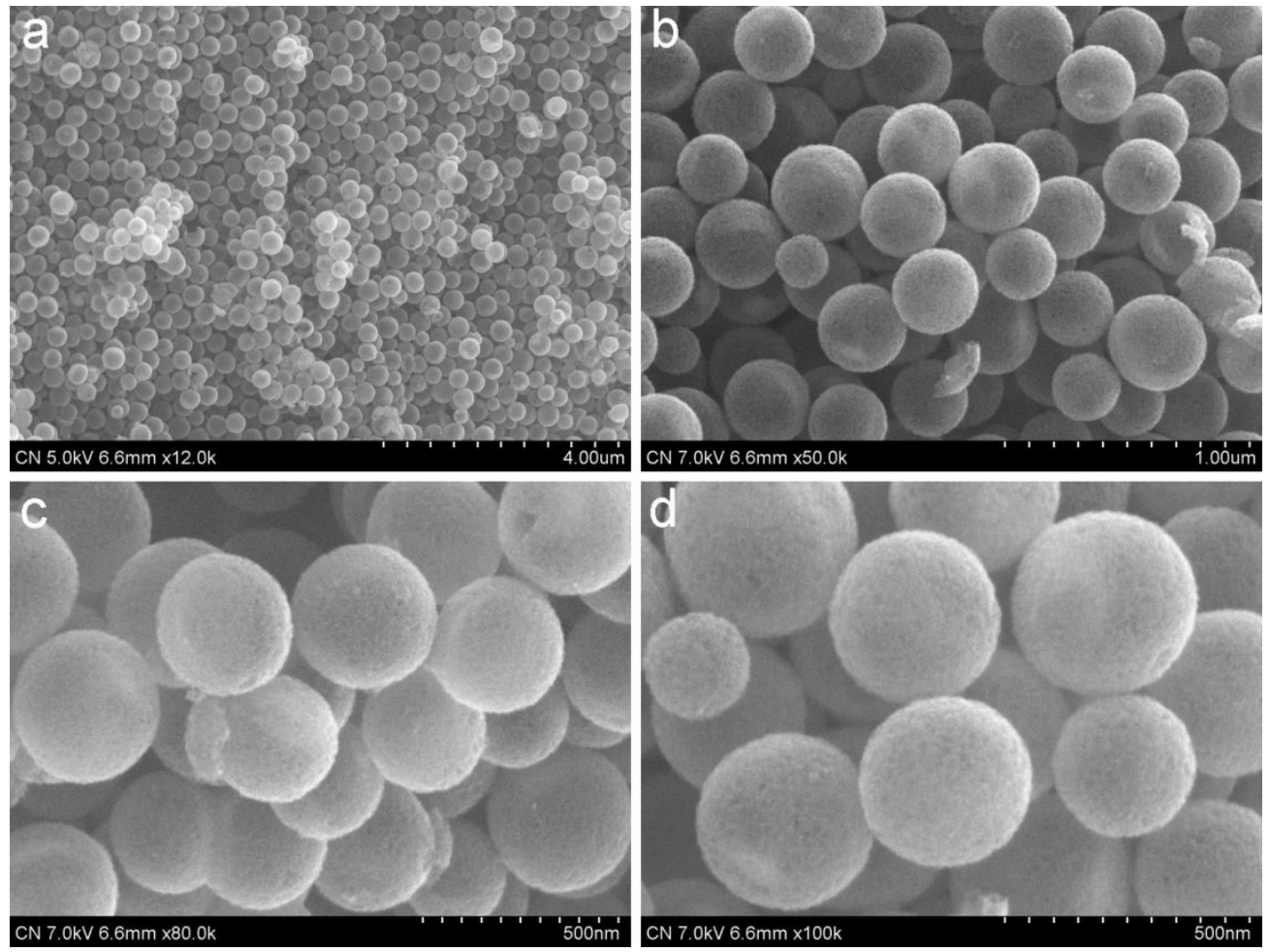

Figure S9. Morphological characterization. a, b, c, d Enlarged SEM images of p-CN@Pt SAs. 

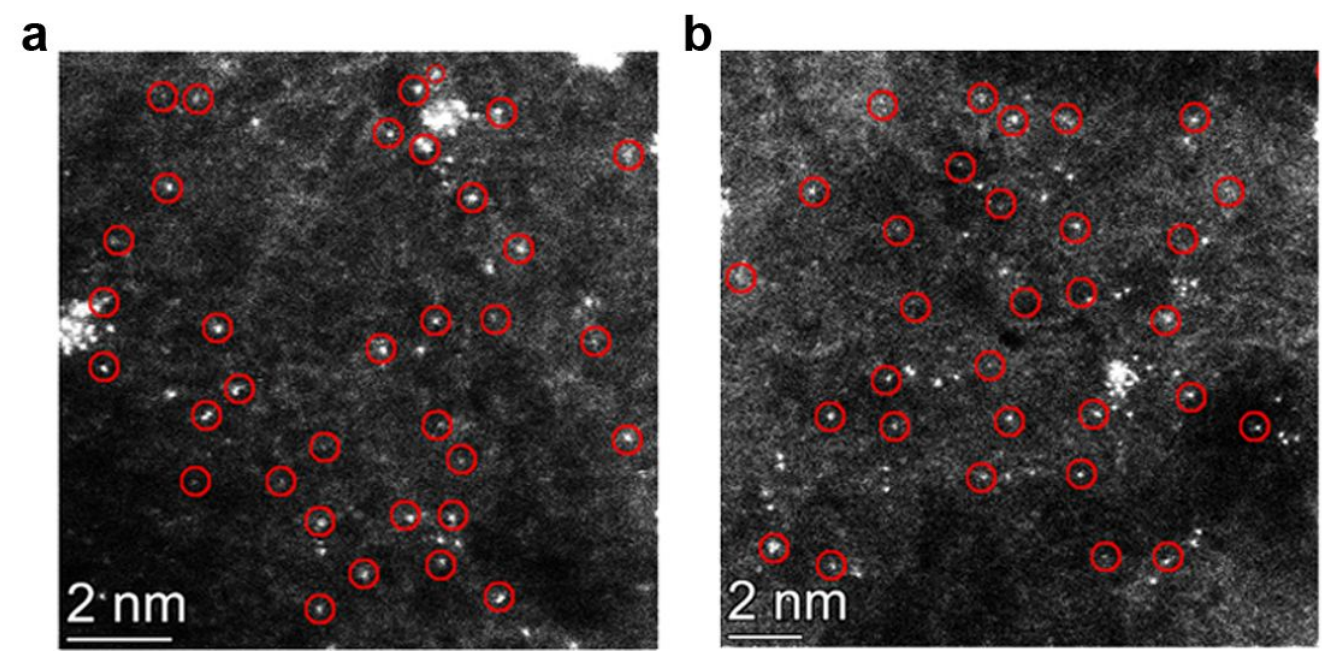

Figure S10. Structural characterizations of $p$-CN@S-Pt. a, b Enlarged STEM images of $p$-CN@S-Pt with the atomic resolution. Some Pt SAs were marked by red cycles. 


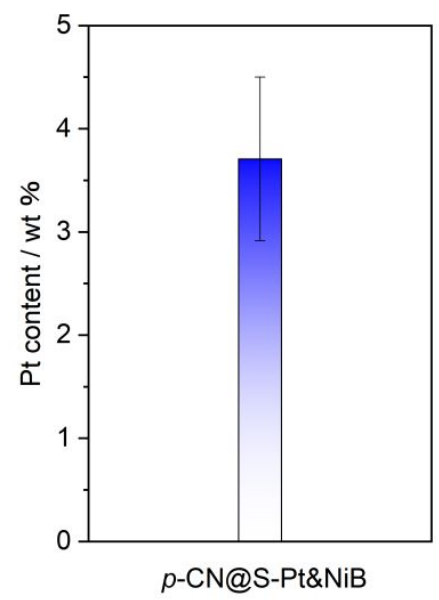

Figure S11. Loading amount of the Pt of the catalysts measured by the ICP-MS. 

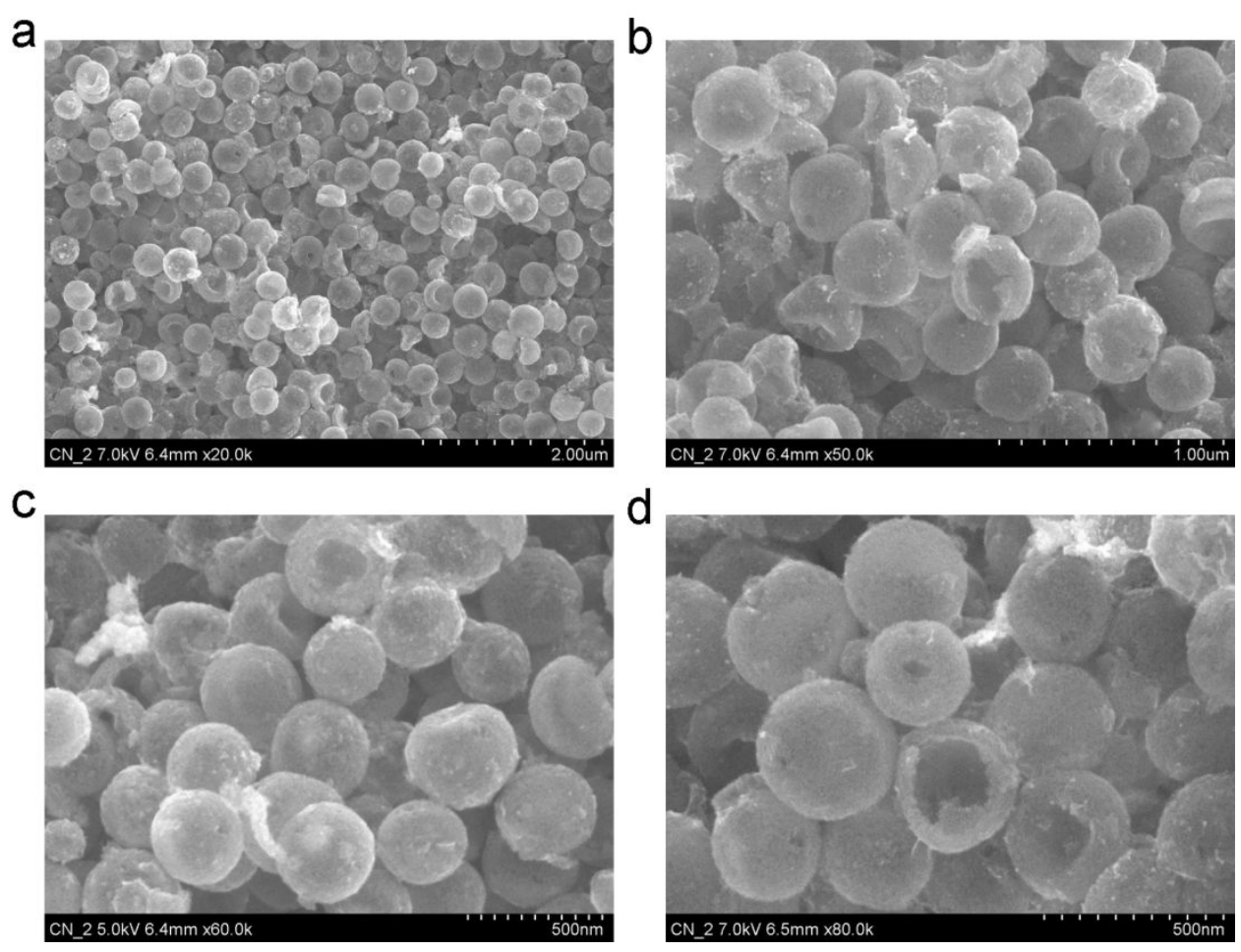

Figure S12. Morphological characterization. a, b, c, d Enlarged SEM images of p-CN@S-Pt\&NiB. 

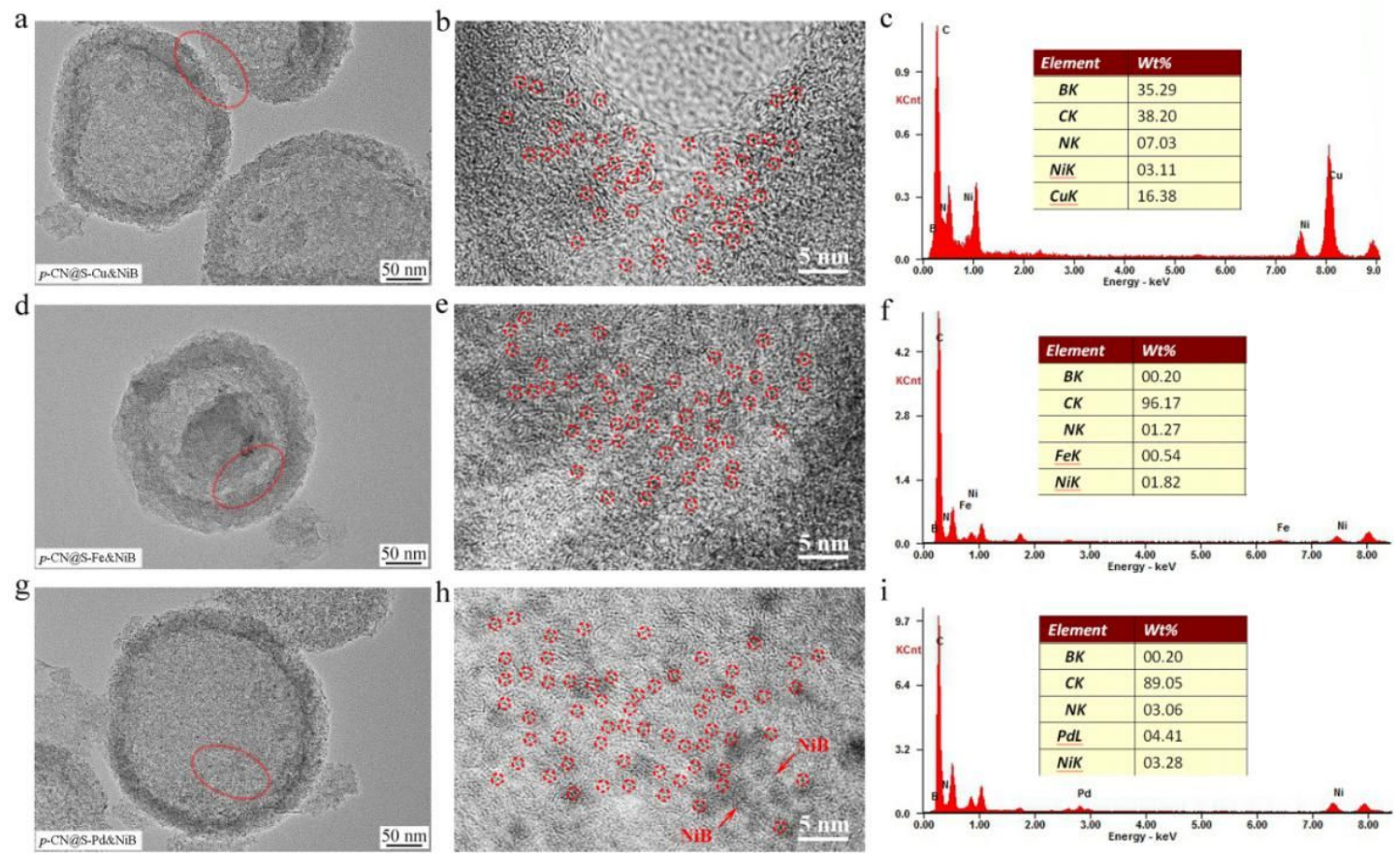

Figure S13. TEM characterization of different metal single atom catalyst. a, b, $\mathbf{c}$ The enlarged HRTEM images and the corresponding EDS results of $\mathrm{Cu}$. d, e, f The HRTEM images and the corresponding EDS results of Fe. $\mathbf{g}, \mathbf{h}, \mathbf{i}$ The HRTEM images and the corresponding EDS results of Pd. 

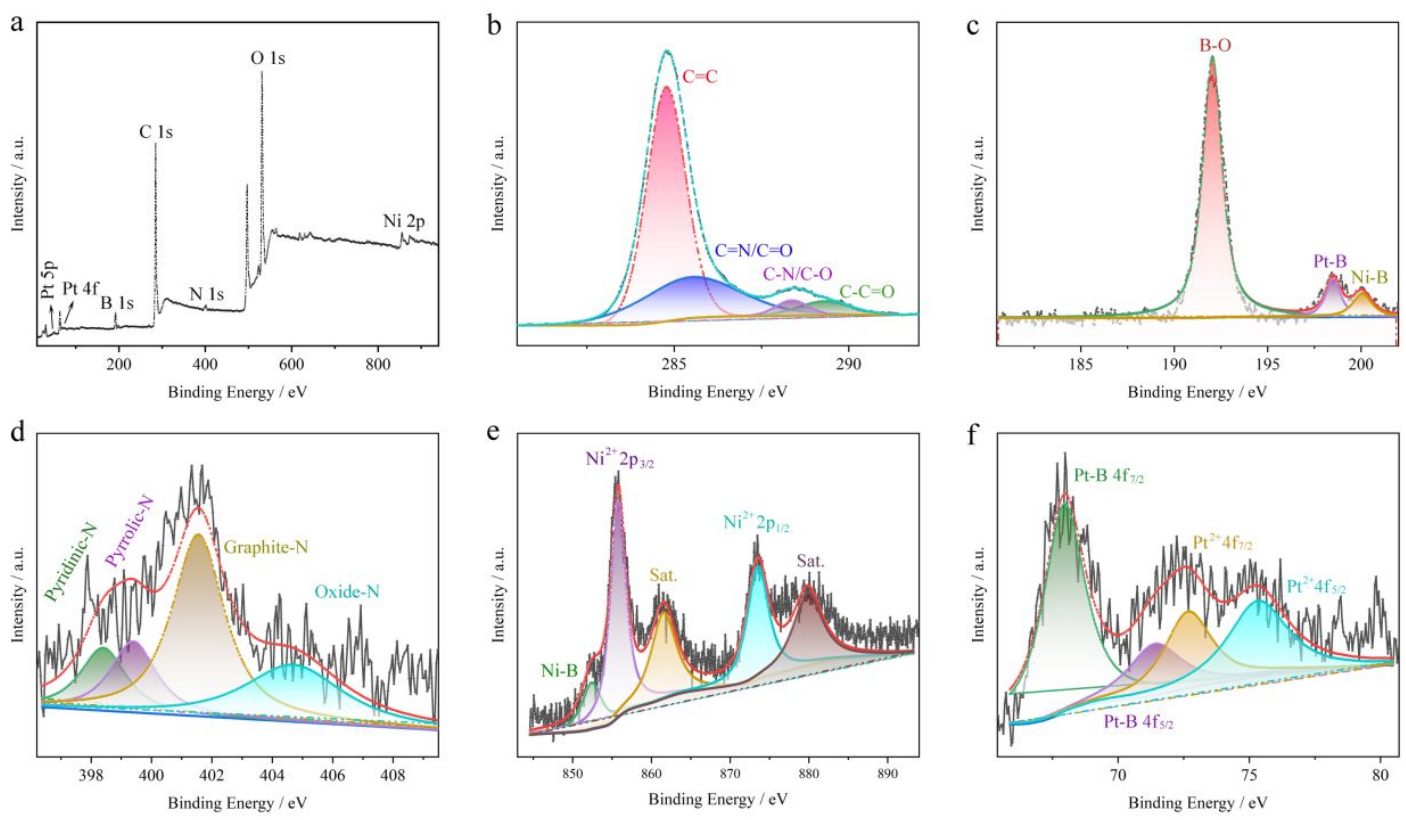

Figure S14. XPS survey pattern of $p$-CN@S-Pt\&NiB. a The XPS of the p-CN@S-Pt\&NiB sample and the high-resolution XPS spectra of b C 1s, c B 1s, d N 1s, e Ni 2p, f Pt 4f. 


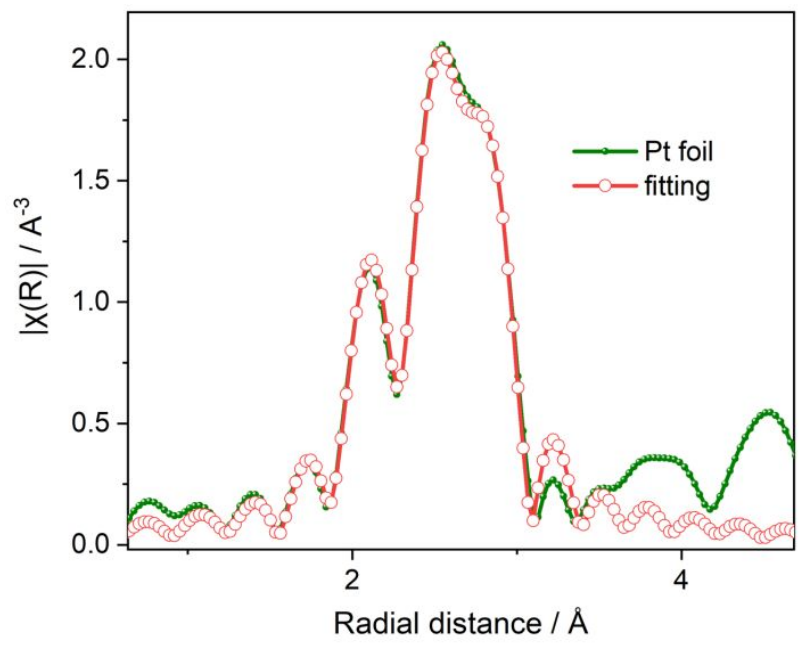

Figure S15. FT-EXAFS fitting curves of $\mathrm{Pt}$ foil at $\mathrm{Pt}_{3}$ edge. 
Table S1. EXAFS parameters of $p$-CN@S-Pt\&NiB.

\begin{tabular}{|c|c|c|c|c|c|}
\hline Sample & bond & $\mathrm{CN}$ & $\mathrm{R}\left(\AA^{2}\right)$ & $\sigma^{2}\left(10^{-3} \AA^{2}\right)$ & $\Delta \mathrm{E}(\mathrm{eV})$ \\
\hline \multirow{2}{*}{ Pt foil } & Pt-Pt & 12 (fixed) & 2.76 & 4.8 & 7.7 \\
\hline \multirow{2}{*}{ Pt-CN } & Pt-N & 4.3 & 2.02 & 1.2 & 1.4 \\
\cline { 2 - 6 } & Pt-Ni & 0.6 & 2.07 & 1.4 & 2.7 \\
\hline
\end{tabular}

Amplitude reduction factor $\left(\mathrm{S}_{0}^{2}=0.85\right.$, based on Pt foil).

$\mathrm{CN}$ is coordination number; $\mathrm{R}$ is bond length; $\sigma^{2}$ is the Debye-Waller factor; $\Delta \mathrm{E}$ is the inner potential correction. Error bounds (accuracies) are estimated as $\mathrm{CN}, \pm 5 \%$; , $\pm 1 \% ; \sigma 2, \pm 1 \% ; \Delta \mathrm{E}, \pm 20 \%$

${ }^{*}$ This value was fixed during EXAFS fitting, based on the known structure of Pt. 

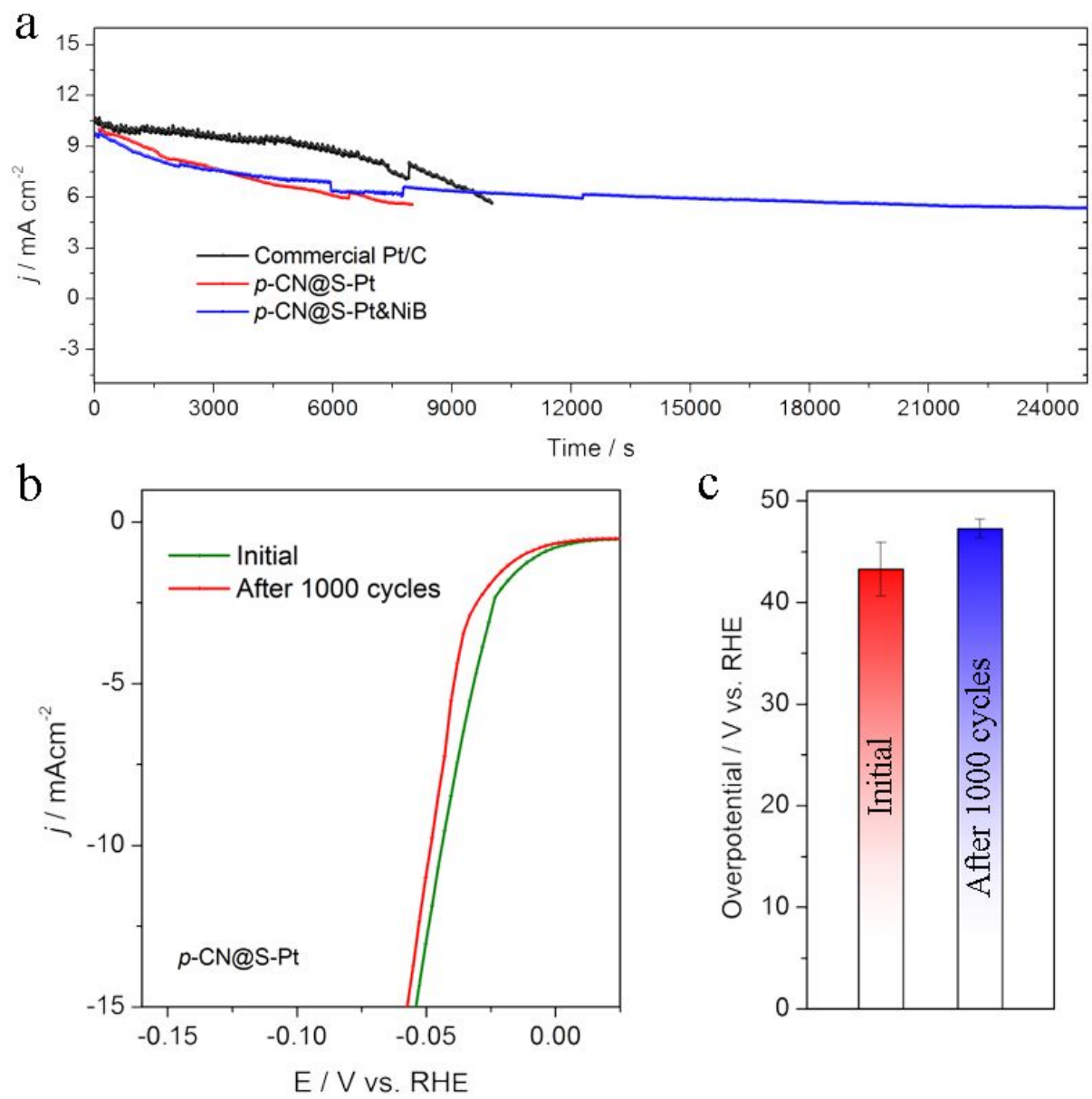

Figure S16. Stability test of the Pt-based catalysts. a Time-dependent current density curve of commercial Pt/C, $p$-CN@S-Pt and p-CN@S-Pt\&NiB at 10 mA cm-2. b Durability test of $p$-CN@S-Pt, the LSV curves measured before and after 1000 cycles test. c The overpotential under $10 \mathrm{mAcm}^{-2}$ before and after stability test. All experiments were done in $0.5 \mathrm{M} \mathrm{H}_{2} \mathrm{SO}_{4}$. 


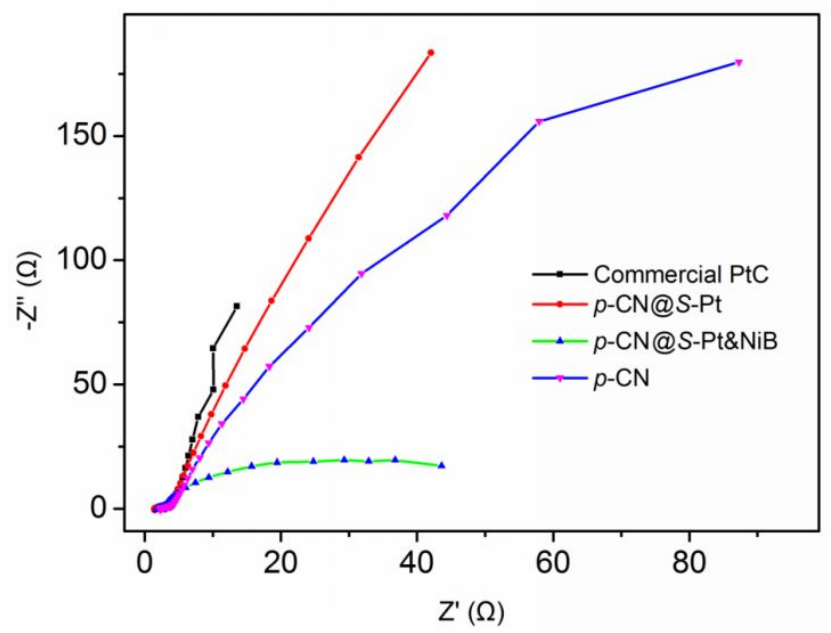

Figure S17. EIS Nyquist plots of commercial Pt/C, p-CN@Pt SAs, p-CN and p-CN@S-Pt\&NiB catalysts. 
Table S2. The comparison of the overpotentials and Tafel plots for HER of reported catalysts recently.

\begin{tabular}{|c|c|c|c|c|}
\hline Catalyst & Tafel $\left(\mathrm{mV} \mathrm{dec}{ }^{-1}\right)$ & Over potential $(\mathrm{mV})$ & Electrolyte & reference \\
\hline $\begin{array}{l}p \text {-CN@S-Pt\&Ni } \\
\text { B }\end{array}$ & 29.26 & 40.6 & $0.5 \mathrm{~m} \mathrm{H}_{2} \mathrm{SO}_{4}$ & This work \\
\hline $\begin{array}{l}\text { Commercial } \\
\mathrm{Pt} / \mathrm{C}\end{array}$ & 46.45 & 50.7 & $0.5 \mathrm{~m} \mathrm{H}_{2} \mathrm{SO}_{4}$ & This work \\
\hline Pt-MoS 2 & 96 & $\sim 145$ & $0.5 \mathrm{~m} \mathrm{H}_{2} \mathrm{SO}_{4}$ & 1 \\
\hline $\mathrm{Pt} / \mathrm{C}(40 \mathrm{wt} \%)$ & 32 & $\sim 70$ & $0.5 \mathrm{~m} \mathrm{H}_{2} \mathrm{SO}_{4}$ & 2 \\
\hline $\mathrm{N}-\mathrm{CoP}_{2}$ & 46 & 38 & $0.5 \mathrm{~m} \mathrm{H}_{2} \mathrm{SO}_{4}$ & 3 \\
\hline $\mathrm{CoP}_{2}$ & 73 & 125 & $0.5 \mathrm{~m} \mathrm{H}_{2} \mathrm{SO}_{4}$ & 3 \\
\hline $\mathrm{Pt} / \mathrm{C}(20 \mathrm{wt} \%)$ & 68 & $\sim 94$ & $\begin{array}{c}0.1 \mathrm{~m} \mathrm{KOH} \\
\text { solution }\end{array}$ & 4 \\
\hline $\mathrm{Mo}_{1} \mathrm{~N}_{1} \mathrm{C}_{2}$ & 90 & 132 & $\begin{array}{c}0.1 \mathrm{~m} \mathrm{KOH} \\
\text { solution }\end{array}$ & 4 \\
\hline $\mathrm{Mo}_{2} \mathrm{C}$ & 102 & 107 & $\begin{array}{c}0.1 \mathrm{~m} \mathrm{KOH} \\
\text { solution }\end{array}$ & 4 \\
\hline $\mathrm{MoN}$ & 163 & 121 & $\begin{array}{c}0.1 \mathrm{~m} \mathrm{KOH} \\
\text { solution }\end{array}$ & 4 \\
\hline
\end{tabular}




\begin{tabular}{|c|c|c|c|c|}
\hline Co-SAC & 99 & 230 & $0.5 \mathrm{~m} \mathrm{H}_{2} \mathrm{SO}_{4}$ & 5 \\
\hline Ni-SAC & 167 & 530 & $0.5 \mathrm{~m} \mathrm{H}_{2} \mathrm{SO}_{4}$ & 5 \\
\hline $\mathrm{Pt} / \mathrm{C}-20 \%$ & 46.2 & 24.1 & $0.5 \mathrm{~m} \mathrm{H}_{2} \mathrm{SO}_{4}$ & 6 \\
\hline $\mathrm{Pt} / \mathrm{C}$ & 43 & 31 & $0.5 \mathrm{~m} \mathrm{H}_{2} \mathrm{SO}_{4}$ & 7 \\
\hline $\mathrm{CoP} / \mathrm{Co}-\mathrm{MOF}$ & 63 & 49 & $0.5 \mathrm{~m} \mathrm{H}_{2} \mathrm{SO}_{4}$ & 7 \\
\hline $\mathrm{CoP}$ & 89 & 97 & $0.5 \mathrm{~m} \mathrm{H}_{2} \mathrm{SO}_{4}$ & 7 \\
\hline $\mathrm{MoS}_{2} / \mathrm{RGO}$ & 41 & 100 & $0.5 \mathrm{~m} \mathrm{H}_{2} \mathrm{SO}_{4}$ & 8 \\
\hline N-P-Ni & 34 & 25.8 & $0.5 \mathrm{~m} \mathrm{H}_{2} \mathrm{SO}_{4}$ & 9 \\
\hline $\mathrm{MoO}_{2} \mathrm{P}_{\mathrm{x}} / \mathrm{Mo}$ & 62 & 135 & $0.5 \mathrm{~m} \mathrm{H}_{2} \mathrm{SO}_{4}$ & 10 \\
\hline $\begin{array}{l}\text { G-C } \mathrm{C}_{3} \mathrm{~N}_{4} @ \mathrm{~N}, \mathrm{P}-\mathrm{do} \\
\text { ped G }\end{array}$ & 90 & 114 & $0.5 \mathrm{~m} \mathrm{H}_{2} \mathrm{SO}_{4}$ & 11 \\
\hline BCN-2-800 & 92 & 208 & $0.5 \mathrm{~m} \mathrm{H}_{2} \mathrm{SO}_{4}$ & 12 \\
\hline $\mathrm{BCN}$ & 100 & 298 & $0.5 \mathrm{~m} \mathrm{H}_{2} \mathrm{SO}_{4}$ & 13 \\
\hline $\mathrm{Pt} @ \mathrm{C}_{2} \mathrm{~N}$ & 33 & $\sim 40$ & $0.5 \mathrm{~m} \mathrm{H}_{2} \mathrm{SO}_{4}$ & 14 \\
\hline $\mathrm{Pd} @ \mathrm{C}_{2} \mathrm{~N}$ & 188 & $\sim 300$ & $0.5 \mathrm{~m} \mathrm{H}_{2} \mathrm{SO}_{4}$ & 14 \\
\hline $\begin{array}{l}\text { Mesoporous } \\
\mathrm{MoS}_{2}\end{array}$ & 50 & 233 & $0.5 \mathrm{~m} \mathrm{H}_{2} \mathrm{SO}_{4}$ & 15 \\
\hline $\mathrm{CoN}_{\mathrm{x}} / \mathrm{C}$ & 57 & 133 & $0.5 \mathrm{~m} \mathrm{H}_{2} \mathrm{SO}_{4}$ & 16 \\
\hline $\begin{array}{l}\mathrm{MoC}_{\mathrm{x}} \\
\text { nanooctahedrons }\end{array}$ & 53 & 142 & $0.5 \mathrm{~m} \mathrm{H}_{2} \mathrm{SO}_{4}$ & 17 \\
\hline
\end{tabular}




\section{Reference}

1. Deng, J.; Li, H.; Xiao, J.; Tu, Y.; Deng, D.; Yang, H.; Tian, H.; Li, J.; Ren, P.; Bao, X. Triggering the Electrocatalytic Hydrogen Evolution Activity of the Inert Two-Dimensional $\mathrm{MoS}_{2}$ Surface via Single-Atom Metal Doping. Energy Environ. Sci. 2015, 8, 1594.

2. Ojani, R., Raoof, J.-B., Hasheminejad, E. Fabrication of a Bimetallic $\mathrm{Cu} / \mathrm{Pt}$ Particle-Modified Carbon Nanotube Paste Electrode and Its Use for the Electrocatalytic Oxidation of Methanol. Int. J. Hydrogen. Energy. 2013, 38, 16394-16401.

3. Cai, J.; Song, Y.; Zang, Y.; Niu, S.; Wu, Y.; Xie, Y.; Zheng, X.; Liu, Y.; Lin, Y.; Liu, X.; Wang, G.; Qian, Y. N-Induced Lattice Contraction Generally Boosts the Hydrogen Evolution Catalysis of P-Rich Metal Phosphides. Sci. Adv. 2020, 6, 8113.

4. Chen, W.; Pei, J.; He, C. T.; Wan, J.; Ren, H.; Zhu, Y.; Wang, Y.; Dong, J.; Tian, S.; Cheong, W. C.; Lu, S.; Zheng, L.; Zheng, X.; Yan, W.; Zhuang, Z.; Chen, C.; Peng, Q.; Wang, D.; Li, Y. Rational Design of Single Molybdenum Atoms Anchored on N-Doped Carbon for Effective Hydrogen Evolution Reaction. Angew. Chem. Int. Ed. 2017, 56, $16086-16090$.

5. Hossain, M. D.; Liu, Z.; Zhuang, M.; Yan, X.; Xu, G.; Gadre, C. A.; Tyagi, A.; Abidi, I. H.; Sun, C.; Wong, H.; Guda, A.; Hao, Y.; Pan, X.; Amine, K.; Luo, Z. 
Rational Design of Graphene-Supported Single Atom Catalysts for Hydrogen Evolution Reaction. Adv. Energy Mater. 2019, 9, 1803689.

6. Wan, X., Wu, H., Guan, B., Luan, D., and Lou, X. Confining Sub-Nanometer Pt Clusters in Hollow Mesoporous Carbon Spheres for Boosting Hydrogen Evolution Activity. Adv. Mater. 2019, 31, 1901349.

7. Liu, T.; Li, P.; Yao, N.; Cheng, G.; Chen, S.; Luo, W.; Yin, Y. CoP-Doped MOF-Based Electrocatalyst for pH-Universal Hydrogen Evolution Reaction. Angew. Chem. Int. Ed. 2019, 58, 4679-4684.

8. Li, Y., Wang, H., Xie, L., Liang, Y., Hong, G. and Dai, H. MoS 2 Nanoparticles Grown On Graphene: An Advanced Catalyst for the Hydrogen Evolution Reaction. $J$. Am. Chem. Soc. 2011, 133, 7296-7299.

9. Jin, H.; Liu, X.; Chen, S.; Vasileff, A.; Li, L.; Jiao, Y.; Song, L.; Zheng, Y.; Qiao, S.-Z. Heteroatom-Doped Transition Metal Electrocatalysts for Hydrogen Evolution Reaction. ACS Energy Lett. 2019, 4, 805-810.

10 Xie, X.; Yu, R.; Xue, N.; Yousaf, A. B.; Du, H.; Liang, K.; Jiang, N.; Xu, A. P Doped Molybdenum Dioxide on Mo Foil with High Electrocatalytic Activity for the Hydrogen Evolution Reaction. J. Mater. Chem. A 2016, 4, 1647.

11. Shinde, S.-S., Sami, A., Lee, J. Nitrogen- and Phosphorus-Doped Nanoporous Graphene/Graphitic Carbon Nitride Hybrids as Efficient Electrocatalysts for Hydrogen Evolution. Chem CatChem 2015, 7, 3873. 
12. Tabassum, H., Zou, R., Mahmood, A., Liang, Z., Guo, S. A Catalyst-Free Synthesis of B, N Co-Doped Graphene Nanostructures with Tunable Dimensions as Highly Efficient Metal Free Dual Electrocatalysts. J. Mater. Chem. A 2016, 4, 16469.

13. Chhetri, M., Maitra, S., Chakraborty, H., Waghmare, U.-V., Rao, C. Superior Performance of Borocarbonitrides, $\mathrm{B}_{\mathrm{x}} \mathrm{C}_{\mathrm{y}} \mathrm{N}_{\mathrm{z}}$, as Stable, Low-Cost Metal-Free Electrocatalysts for the Hydrogen Evolution Reaction. Energy Environ. Sci. 2016, 9, 95.

14. Mahmood, J.; Li, F.; Jung, S.; Okyay, M.; Ahmad, I.; Kim, S.; Park, N.; Young, H.; Baek, J. An Efficient and pH-Universal Ruthenium-Based Catalyst for the Hydrogen Evolution Reaction. Nat. Nanotechnology 2017, 12, 441-446.

15. Kibsgaard, J., Chen, Z., Reinecke B., Jaramillo, T. Engineering the Surface Structure of $\mathrm{MoS}_{2}$ to Preferentially Expose Active Edge Sites for Electrocatalysis. Nat. Mater. 2012, 11, 963-969.

16. Liang, H.; Bruller, S.; Dong, R.; Zhang, J.; Feng, X.; Müllen, K. Molecular Metal- $\mathrm{N}_{\mathrm{x}}$ Centres in Porous Carbon for Electrocatalytic Hydrogen Evolution. Nat. Comm. 2015, 6, 7992.

17. Wu, H.; Xia, B.; Yu, L.; Yu X.; Lou. X. Porous Molybdenum Carbide Nano-Octahedrons Synthesized via Confined Carburization in Metal-Organic Frameworks for Efficient Hydrogen Production. Nat. Comm. 2015, 6, 6512. 